\title{
CalCannabis: Regulating a previously unregulated industry
}

\section{An interview with Richard Parrott, Director of the California Department of Food and Agriculture's CalCannabis Cultivation Licensing Division}

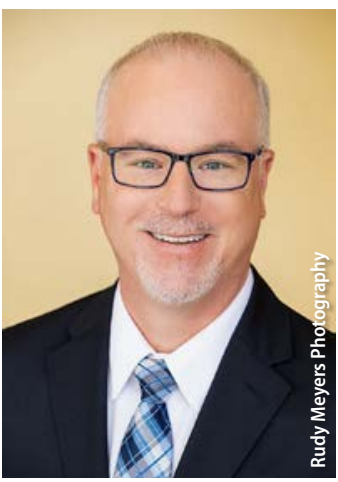

Richard Parrott
Online: https://doi.org/10.3733/ ca.2019a0020

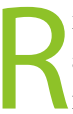
ichard Parrott directs CalCannabis, the state agency that licenses commercial cannabis farmers and oversees the California Cannabis Track-and-Trace system, which tracks all commercial cannabis and cannabis products - from cultivation to sale. He has served in state government for more than three decades, primarily at the State Board of Equalization - where he administered 30 tax and fee programs, including a program focused on alcoholic beverage taxes.

\section{Could you please briefly explain what CalCannabis does?}

Yes. CalCannabis Cultivation Licensing is a division within the California Department of Food and Agriculture. We license all cannabis cultivation for the state of California. We are also the agency responsible for leading the implementation of the statewide cannabis track-and-trace system.

We have two branches within our division. Our licensing branch is responsible for issuing and renewing licenses for all the cultivators in California. Our compliance and enforcement branch contains the team that implements track-and-trace and also our field staff - special investigators who go to licensed sites and perform inspections to make sure farmers are complying with all state rules. They are limited peace officers - they have powers of arrest and the ability to obtain search warrants, but they are not armed. At CalCannabis we work very closely with our partner agencies out in the field, such as the California Department of Fish and Wildlife, the State Water Resources Control Board, the Bureau of Cannabis Control and state law enforcement.

Since CalCannabis focuses mainly on cultivation, how does it work institutionally for you to track and trace cannabis all the way to the point of retail sale?

Although we're the agency responsible for leading the implementation of the track-and-trace system, there are three state agencies that license cannabis businesses in California: the California Department of Food and Agriculture for cultivation; the California Department of Public Health for manufacturers of products such as edibles; and the Bureau of Cannabis Control for testing labs, retailers, distributors, temporary cannabis events and microbusinesses (a microbusiness licensee is allowed to engage in at least three smaller-scale commercial cannabis activities at once, such as cultivation, manufacturing, distribution and/or retail). We've worked very closely with our partner agencies on the implementation of track-and-trace. We have been meeting with teams from those agencies since 2016 to make sure all of the agencies' requirements are captured within the track-and-trace system. Every licensee is required to use the track-and-trace system. Cultivators affix identification tags to their plants and, as they harvest those plants and package flower, the packages will have identification tags. As products go to manufacturers who perform extraction processes, the extracted product will go into containers that have tags. Essentially the concept of the track-and-trace system is that all products are tagged from cultivation until they work their way through to retail. Every time the product changes hands, every time it moves from one licensee to another, or changes its composition, everything is tracked and accounted for.

\section{But how is enforcement of track-and-trace handled? Who in particular makes sure that people all through the system comply with the tagging and reporting requirements?}

We work with the Bureau of Cannabis Control and the California Department of Public Health to ensure everyone is trained and knows what's in the system. When the three agencies go out and do inspections - for example, when we at CalCannabis inspect a cultivation site - we look at the system and it tells us how many plants that cultivator has tagged, and we'll verify that. Each agency has its own compliance and enforcement team. Everyone uses track-and-trace as a component of their inspections. It's basically a tool for us to determine compliance. It's not the only thing we use, but it's definitely a tool that helps us gauge whether a licensee is doing things correctly.

Now, medicinal and adult-use cannabis are legal in California, but still illegal under federal law. What challenges do you encounter in overseeing the cultivation of a crop with an ambiguous legal status?

Certainly there are challenges. Prior to this position, I worked for 30 years for the California Department 
of Tax and Fee Administration, and oversaw a lot of tax and fee programs where there was a coordinated state and federal system. For example, I worked for the Board of Equalization on their alcoholic beverage tax program. There was a very coordinated sync-up between state and federal regulations for alcohol and for reporting tax collection at the federal level and the state level - and you could always look to federal guidelines or regulations. We're not able to do that here. It's just not as smooth. I could give you a couple of examples one of them is banking. Cultivators pay license fees to us, but those who can't engage in banking [because of federal restrictions] have to pay their fees in cash. We have arrangements so they can make cash payments in Sacramento or Eureka. We have a contract with the Bureau of Cannabis Control so our applicants can make an appointment with the Bureau in Sacramento and pay cash. Or, if they're in a northern county, we have an office in Eureka.

Another example is pesticides. Pesticide labels have to be approved at the federal level. [Because cannabis is illegal at the federal level], no pesticides are approved for use on cannabis. The Bureau of Cannabis Control has regulations on the amount of a [particular] pesticide that would trigger a [cannabis] product to fail testing, and we work very closely with the California Department of Pesticide Regulation, and with county agricultural commissioners, on which pesticides can be used. But if you had a coordinated federal system, it would be very clear which pesticides could be used. You would have the federal Environmental Protection Agency saying, "These are okay. These are legal for use."

Growers sometimes complain that the burdens of achieving compliance with cannabis regulations are very high. Then again, I've heard the argument that cannabis regulations are actually less onerous than forestry regulations. What's your response to the growers' viewpoint, as well as to this alternative viewpoint?

There are always different viewpoints. And although cannabis cultivation has been happening for a very long time in California, state and local regulation is new for the industry. The existing industry has been used to doing things a certain way. And then you go from zero regulation to all kinds of regulation, almost overnight. So there are understandably a lot of growing pains, as there will be with any new program. I always stress that we see this as a partnership with the industry. We did a lot of stakeholder outreach across the state as we developed the regulations. Certain procedures were mandated by statute, and we created others through our regulatory process. We carefully considered all public input and, where we could make changes along the way, we have. And we're going to continue to engage with the industry.

We also have two new programs on the horizon. One is a comparable-to-organic program for cannabis, called OCal, and we are creating a process for cultivators to establish appellations for cannabis, called the CalCannabis Appellations Project. Those programs are mandated by law and must be in place by January 1, 2021.

\section{Why "comparable-to-organic" instead of just "organic"?}

Interesting you say that. I can point you back to the [previous] question on the conflict [between federal and state cannabis law]. Under the U.S. Department of Agriculture (USDA) is the National Organic Program. The state laws that were passed for legalization of both medicinal and adult-use cannabis say that the state will create an organic program for cannabis that's comparable to the National Organic Program - and the reason we call it "comparable" instead of "organic" is because the term "organic" is basically owned by the USDA and the National Organic Program. So, while we will follow the same path that's being used [at the federal level], meaning the same guidelines, we just can't call it "organic" until or unless there is a sync-up between federal and state laws.

I'm told by some enthusiasts of sun-grown cannabis that it's allowed in relatively few California localities - but indoor cultivation, on the other hand, requires a lot of electricity, which sun-grown cannabis growers will tell you conflicts with the state's climate goals. Does CalCannabis have a position on this one way or another? And if you favor sun-grown cannabis, what can you do to encourage it, if anything?

As a regulatory agency, we don't take a position on or favor any particular part of the industry. Our goal is to have regulations that we apply consistently across the licensee base. We prepared an environmental impact report as we were creating the regulations for our program, and our regulations incorporate greenhouse-gas emission reduction standards that go into effect in 2022. Also, you may not know that we offer 17 types of commercial cannabis cultivation licenses. They are categorized primarily by size and the type of growing process - indoor, outdoor or mixed light.

A serious obstacle to getting full compliance among growers in California is that so much California cannabis goes out of state to places where it's illegal - illegal at the federal level in every state, and illegal on the state level in most states. That's a hard problem. How do you begin to address it?

Good question. Difficult issue. Ideally, it would be accomplished at the federal level. And until then, we're working hard every day to bring people into the regulated market. We're issuing licenses every day and we're processing applications every day. And when we're aware of people who aren't getting into the [regulated] market and are creating an unfair marketplace, we work as diligently as we can with our partner agencies to address those issues. It's not going to happen overnight. [As we continue issuing licenses,] we are ramping up and filling all our field enforcement positions so we will have staff located statewide to conduct inspections at licensed sites and work with our partner agencies on addressing [unlicensed cultivation]. We also work to ensure that, if a site is licensed, [other agencies] are aware of that. We're here to protect the people who are licensed, and part of that protection is addressing those who aren't licensed, because that creates an unfair marketplace. And by licensing and regulating commercial cannabis farmers in California, we're also ensuring public safety and environmental protection. CA

For more information about CalCannabis Cultivation Licensing, a division of the California Department of Food and Agriculture, visit calcannabis.cdfa.ca.gov. 